\title{
Isomorphism problem in a special class of Banach function algebras and its application
}

\author{
Sin-EI TAKAHASI, KiYOSHI SHIRAYANAGI*, AND MAKOTO TSUKADA
}

\begin{abstract}
Given a weight function $\tau$, we introduce a new class of Banach function algebras with respect to $\tau$, denoted by $C_{0 b}(X, \tau)$. We provide a complete solution to the isomorphism problem in this class. We further characterize the BSE-extension and the Inoue-Doss ideal associated with it. As an application of our results, we show the equivalence of the four statements: (i) $C_{0 b}(X, \tau)$ is of BSE, (ii) $C_{0 b}(X, \tau)$ is of $\mathrm{BED}$, (iii) $C_{0 b}(X, \tau)$ is Tauberian and (iv) $\tau$ is bounded.
\end{abstract}

Keywords: Banach function algebra of type I, BSE-extension, Inoue-Doss ideal, BSE-algebra, BED-algebra, Tauberian Banach algebra.

2020 Mathematics Subject Classification: Primary 46J10; Secondary 46J40, 43A25.

\section{Dedicated to the memory of Professor Wataru Takahashi.}

\section{INTRODUCTION AND MAIN RESULTS}

Let $X$ be a locally compact Hausdorff space and $C_{b}(X)$ be the Banach algebra of all bounded complex-valued continuous functions on $X$ with supremum norm $\|\cdot\|_{\infty}$. Define $C_{0}(X)=\{f \in$ $C_{b}(X): f$ vanishes at infinity $\}$. Let $\tau$ be a positive continuous function on $X$ with $\inf _{x \in X} \tau(x) \geq$ 1. Define

$$
\begin{aligned}
& C_{00}(X, \tau)=\left\{f \in C_{0}(X): f \tau \in C_{0}(X)\right\}, \\
& C_{0 b}(X, \tau)=\left\{f \in C_{0}(X): f \tau \in C_{b}(X)\right\}, \\
& C_{b b}(X, \tau)=\left\{f \in C_{b}(X): f \tau \in C_{b}(X)\right\}
\end{aligned}
$$

and

$$
\|f\|_{\infty, \tau}=\sup _{x \in X}|f(x)| \tau(x) \quad\left(f \in C_{b}(X)\right) .
$$

Then both $C_{00}(X, \tau)$ and $C_{0 b}(X, \tau)$ are subalgebras of $C_{0}(X)$, and $C_{b b}(X, \tau)$ is a subalgebra of $C_{b}(X)$. Moreover, these algebras become Banach algebras with norm $\|\cdot\|_{\infty, \tau}$, and they have the inclusion relation

$$
C_{c}(X) \subseteq C_{00}(X, \tau) \subseteq C_{0 b}(X, \tau) \subseteq C_{b b}(X, \tau) \subseteq C_{b}(X)
$$

where $C_{c}(X)$ is the set of all complex-valued continuous functions on $X$ with compact supports.

Remark 1.1. Put $C_{b 0}(X, \tau)=\left\{f \in C_{b}(X): f \tau \in C_{0}(X)\right\}$. Then, it follows that $C_{00}(X, \tau)=$ $C_{b 0}(X, \tau)$ holds. 
Note that $\tau$ is a $C_{0}(X)$-local function on $X$, and hence the algebra $C_{0}(X)_{\tau(1)}$ is defined (see [5, Definitions 5.1 and 5.3] for definition). Since $C_{0}(X)_{\tau(1)}=C_{00}(X, \tau)$, it follows from [5, Theorem 5.4 (ii)] that $C_{00}(X, \tau)$ is a Segal algebra in $C_{0}(X)$ with norm $\|\cdot\|_{\infty, \tau}$, and hence its Gelfand space can be identified with $X$ (see [5, Theorem B']). Moreover, $C_{00}(X, \tau)$ is always a BED-algebra of type I, but it is not a BSE-algebra if $\tau$ is unbounded (see [2, Theorem 6.2]). On the other hand, $C_{0 b}(X, \tau)$ is generally not a Segal algebra in $C_{0}(X)$. However, J. Inoue et al. have shown that $C_{0 b}(X, \tau)$ is a Banach algebra of type I but is neither Tauberian nor of BSE nor of BED if $1 / \tau$ vanishes at infinity (see [2, Theorem 7.3]). The aim of this paper is to investigate this algebra in greater detail. We refer the reader to $[4,5,6,9,10,11,13]$ for more details on Segal algebras, BSE-algebras, BED-algebras and type I Banach algebras.

We first give a complete solution to the isomorphism problem in $C_{0 b}(X, \tau)$. To state this, let $Y$ be another locally compact Hausdorff space and $\sigma$ be another positive continuous function on $Y$ with $\inf _{y \in Y} \sigma(y) \geq 1$. Then we have:

Theorem 1.1. The following three statements are equivalent:

(i) $C_{00}(X, \tau)$ is isomorphic to $C_{00}(Y, \sigma)$.

(ii) $C_{0 b}(X, \tau)$ is isomorphic to $C_{0 b}(Y, \sigma)$.

(iii) There exists a homeomorphism $\eta$ from $Y$ onto $X$ such that $m \sigma \leq \tau \circ \eta \leq M \sigma$ for some positive constants $m$ and $M$.

Next we have:

Theorem 1.2. The following two statements are equivalent:

(i) $C_{00}(X, \tau)$ is isomorphic to $C_{0 b}(Y, \sigma)$.

(ii) Both $X$ and $Y$ are homeomorphic and both $\tau$ and $\sigma$ are bounded.

Moreover, we show that the BSE-extension and the Inoue-Doss ideal associated with $C_{0 b}(X, \tau)$ are equal to $C_{b b}(X, \tau)$ and $C_{00}(X, \tau)$, respectively. The details will be described in Section 5 .

As an application of the above results, we show the following result which is a generalization of [2, Theorem 7.3].

Theorem 1.3. The following five statements are equivalent:

(i) $C_{0 b}(X, \tau)$ is of $B S E$.

(ii) $C_{0 b}(X, \tau)$ is of BED.

(iii) $C_{0 b}(X, \tau)$ is Tauberian.

(iv) $C_{0 b}(X, \tau)$ has a bounded $X$-weak approximate identity.

(v) $\tau$ is bounded.

\section{PRELIMINARIES}

In what follows, let $X$ be a locally compact Hausdorff space and $\tau$ be a positive continuous function on $X$ with

$$
\inf _{x \in X} \tau(x) \geq 1
$$

Let $A$ be a natural Banach function algebra on $X$. Then the natural embedding $\delta_{X}$ from $X$ to the Gelfand space $\Phi_{A}$ of $A$ is surjective, and hence $\delta_{X}$ is homeomorphic by [12, Theorem 3.2.4]. Thus we may identify $\Phi_{A}$ with $X$ if it will cause no confusion. Then the multiplier algebra $M(A)$ of $A$ is described as $\left\{f \in C_{b}(X): f g \in A(g \in A)\right\}$. We say that $A$ is of type I if $M(A)=C_{b}(X)$. We denote by $\operatorname{span}(X)$ the linear span of $X$ in the dual space $A^{*}$ of $A$. Therefore, an arbitrary element $p$ in $\operatorname{span}(X)$ has the unique expression

$$
p=\sum_{x \in X} \widehat{p}(x) x,
$$


where $\widehat{p}$ is a complex-valued function on $X$ with finite support. A function $f \in C_{b}(X)$ is said to be a $B S E$-function associated with $A$ if there exists a constant $\beta>0$ such that

$$
\left|\sum_{x \in X} \widehat{p}(x) f(x)\right| \leq \beta\|p\|_{A^{*}}
$$

for all $p \in \operatorname{span}(X)$. The BSE-norm of $f$, denoted by $\|f\|_{B S E(A)}$, is the infimum of all such $\beta$.

Let $C_{B S E(A)}(X)$ be the set of all BSE-functions on $X$ associated with $A$. Then it is a semisimple commutative Banach algebra with the BSE-norm (see [13, Lemma 1]).

Definition 2.1. We refer to $C_{B S E(A)}(X)$ as the BSE-extension associated with $A$.

An algebra $A$ is said to be a BSE-algebra if $M(A)=C_{B S E(A)}(X)$ (see [13, p.151, Definition]). If $\left\{e_{\lambda}\right\}$ is a net in $A$ satisfying the condition

$$
\lim _{\lambda} e_{\lambda}(x)=1 \quad(x \in X),
$$

then we call it a $X$-weak approximate identity of $A$. We note that $M(A) \subseteq C_{B S E(A)}(X)$ if and only if $A$ has a bounded $X$-weak approximate identity (see [13, Corollary 5]). For the details on $X$-weak approximation identity, refer to $[3,8]$.

Let $\mathcal{K}(X)$ be the directed set consisting of all compact subsets of $X$ with respect to the inclusion order. For $f \in C_{B S E(A)}(X)$ and $K \in \mathcal{K}(X)$, define

$$
\|f\|_{B S E(A), K}=\sup \left\{\left|\sum_{x \in X \backslash K} \widehat{p}(x) f(x)\right|: p \in \operatorname{span}(X),\|p\|_{A^{*}} \leq 1\right\},
$$

and put

$$
C_{B S E(A)}^{0}(X)=\left\{f \in C_{B S E(A)}(X): \lim _{K \in \mathcal{K}(X)}\|f\|_{B S E(A), K}=0\right\} .
$$

Then $C_{B S E(A)}^{0}(X)$ is a closed ideal of $C_{B S E(A)}(X)$ (see [4, Corollary 3.9]). This is an important ideal in our argument.

Definition 2.2. We refer to $C_{B S E(A)}^{0}(X)$ as the Inoue-Doss ideal ${ }^{1}$ associated with $A$.

An algebra $A$ is said to be a BED-algebra if $A=C_{B S E(A)}^{0}(X)$ (see [4, Definition 4.13]). A Banach function algebra $B$ on $X$ is called a Banach ideal of $C_{0}(X)$ if $B$ is an ideal of $C_{0}(X)$ and $\|f g\|_{B} \leq\|f\|_{\infty}\|g\|_{B}$ holds for all $f \in C_{0}(X)$ and $g \in B$ (see [5, Definition 3.1]).

Lemma 2.1. The algebra $C_{0 b}(X, \tau)$ is a dense natural Banach ideal in $C_{0}(X)$.

Proof. It is clear that $C_{0 b}(X, \tau)$ is a dense Banach ideal in $C_{0}(X)$. Hence it suffices to show that $C_{0 b}(X, \tau)$ is natural, that is, the natural embedding $\delta_{X}$ from $X$ to $\Phi_{C_{0 b}(X, \tau)}$ is surjective. To do this, let $\varphi$ be an arbitrary element of $\Phi_{C_{0 b}(X, \tau)}$. Take $h \in C_{0 b}(X, \tau)$ with $\varphi(h) \neq 0$, and define

$$
\widetilde{\varphi}(f)=\varphi(f h) / \varphi(h)\left(f \in C_{0}(X)\right) .
$$

This is well-defined because the right hand side of the above equation is independent of the choice of $h \in C_{0 b}(X, \tau)$ with $\varphi(h) \neq 0$. By an easy calculation, we see that $\widetilde{\varphi} \in \Phi_{C_{0}(X)}$ with $\left.\widetilde{\varphi}\right|_{C_{0 b}(X, \tau)}=\varphi$, and hence there exists $x \in X$ such that $\delta_{X}(x)=\varphi$, namely, $\delta_{X}$ is surjective, as required.

By Lemma 2.1, $\Phi_{C_{0 b}(X, \tau)}$ can be identified with $X$ under the natural embedding.

\footnotetext{
${ }^{1}$ The first author personally learned this important ideal from Professor Jyunji Inoue in the old days. The ideal was first introduced by him, but the underlying idea behind it had appeared in R. Doss [1].
} 
Lemma 2.2. The algebra $C_{0 b}(X, \tau)$ is of type $I$.

Proof. Since $\Phi_{C_{0 b}}(X, \tau)$ can be identified with $X$, it follows that $M\left(C_{0 b}(X, \tau)\right) \subseteq C_{b}(X)$. Also, since $C_{0 b}(X, \tau)$ is an ideal of $C_{b}(X)$, it follows that $C_{b}(X) \subseteq M\left(C_{0 b}(X, \tau)\right)$. Thus we obtain $M\left(C_{0 b}(X, \tau)\right)=C_{b}(X)$, that is, $C_{0 b}(X, \tau)$ is of type I.

\section{PROOF OF THEOREM 1.1}

Let $Y$ be another locally compact Hausdorff space and $\sigma$ be another positive continuous function on $Y$ with $\inf _{y \in Y} \sigma(y) \geq 1$.

(i) $\Rightarrow$ (iii) Suppose that there is an isomorphism $\rho$ from $C_{00}(X, \tau)$ onto $C_{00}(Y, \sigma)$. Then there are positive constants $m, M$ such that

$$
m\|\rho(f)\|_{\infty, \sigma} \leq\|f\|_{\infty, \tau} \leq M\|\rho(f)\|_{\infty, \sigma}\left(f \in C_{00}(X, \tau)\right) .
$$

Let $\rho^{*}$ be the dual map of $\rho$ from $C_{00}(Y, \sigma)^{*}$ onto $C_{00}(X, \tau)^{*}$. Then we have $\rho^{*}\left(\Phi_{C_{00}(Y, \sigma)}\right)=$ $\Phi_{C_{00}(X, \tau)}$. Let $\delta_{X}$ and $\delta_{Y}$ be the natural embedding from $X$ onto $\Phi_{C_{00}(X, \tau)}$ and the natural embedding from $Y$ onto $\Phi_{C_{00}(Y, \sigma)}$, respectively. Define

$$
\eta=\left.\left(\delta_{X}\right)^{-1} \circ \rho^{*}\right|_{\Phi_{C_{00}(Y, \sigma)}} \circ \delta_{Y} .
$$

Then $\eta$ is a homeomorphism from $Y$ onto $X$. We shall show that

$$
\rho(f)=f \circ \eta\left(f \in C_{00}(X, \tau)\right) .
$$

In fact, let us take $f \in C_{00}(X, \tau)$ arbitrarily. Then we have

$$
\begin{aligned}
\rho(f)(y) & =\left\langle\rho(f), \delta_{Y}(y)\right\rangle=\left\langle f, \rho^{*}\left(\delta_{Y}(y)\right)\right\rangle=\left\langle f,\left.\rho^{*}\right|_{\Phi_{C_{00}(Y, \sigma)}}\left(\delta_{Y}(y)\right)\right\rangle \\
& =\left\langle f, \delta_{X}\left\{\delta_{X}^{-1}\left(\left.\rho^{*}\right|_{\Phi_{C_{00}(Y, \sigma)}}\left(\delta_{Y}(y)\right)\right)\right\}\right\rangle \\
& =\left\langle f, \delta_{X}(\eta(y))\right\rangle=f(\eta(y)) \\
& =(f \circ \eta)(y)
\end{aligned}
$$

for all $y \in Y$, that is, (3.2) holds as required. By (3.2), $f \circ \eta \in C_{00}(Y, \sigma)$ holds for all $f \in C_{00}(X, \tau)$.

It remains to show that $m \sigma \leq \tau \circ \eta \leq M \sigma$. To show this, let us take $y \in Y$ and $\varepsilon>0$ arbitrarily. Since $\tau$ is continuous, there exists a neighbourhood $U$ of $\eta(y)$ such that $|\tau(x)-\tau(\eta(y))|<$ $\varepsilon$ for all $x \in U$. Also since $\sigma$ is continuous, there exists a neighbourhood $V$ of $y$ such that $\left|\sigma\left(y^{\prime}\right)-\sigma(y)\right|<\varepsilon$ for all $y^{\prime} \in V$. Put $W=U \cap \eta(V)$. Then $W$ is a neighbourhood of $\eta(y)$. Take a function $f_{0} \in C_{c}(X)$ such that $f_{0}(\eta(y))=1,\left.f_{0}\right|_{X \backslash W}=0$ and $0 \leq f_{0} \leq 1$. By (3.1) and (3.2), we have

$$
m\left\|f_{0} \circ \eta\right\|_{\infty, \sigma} \leq\left\|f_{0}\right\|_{\infty, \tau} \leq M\left\|f_{0} \circ \eta\right\|_{\infty, \sigma} .
$$

Therefore, it follows from the first half of (3.3) that

$$
\begin{aligned}
m \sigma(y) & =m\left|f_{0}(\eta(y))\right| \sigma(y) \leq m\left\|f_{0} \circ \eta\right\|_{\infty, \sigma} \leq\left\|f_{0}\right\|_{\infty, \tau} \\
& =\sup _{x \in X}\left|f_{0}(x)\right| \tau(x)=\sup _{x \in W}\left|f_{0}(x)\right| \tau(x) \leq \sup _{x \in U} \tau(x) \\
& \leq \tau(\eta(y))+\varepsilon .
\end{aligned}
$$


Since $y \in Y$ and $\varepsilon>0$ are arbitrary, we have $m \sigma \leq \tau \circ \eta$. Moreover, it follows from the latter half of (3.3) that

$$
\begin{aligned}
(\tau \circ \eta)(y) & =\tau(\eta(y))=\left|f_{0}(\eta(y))\right| \tau(\eta(y)) \leq\left\|f_{0}\right\|_{\infty, \tau} \\
& \leq M\left\|f_{0} \circ \eta\right\|_{\infty, \sigma}=M \sup _{y^{\prime} \in Y}\left|f_{0}\left(\eta\left(y^{\prime}\right)\right)\right| \sigma\left(y^{\prime}\right) \\
& =M \sup _{y^{\prime} \in \eta^{-1}(W)}\left|f_{0}\left(\eta\left(y^{\prime}\right)\right)\right| \sigma\left(y^{\prime}\right) \leq M \sup _{y^{\prime} \in \eta^{-1}(W)} \sigma\left(y^{\prime}\right) \\
& \leq M \sup _{y^{\prime} \in V} \sigma\left(y^{\prime}\right) \leq M(\sigma(y)+\varepsilon) .
\end{aligned}
$$

Since $y \in Y$ and $\varepsilon>0$ are arbitrary, we have $\tau \circ \eta \leq M \sigma$.

(iii) $\Rightarrow$ (i) Suppose that there is a homeomorphism $\eta$ from $Y$ onto $X$ such that $m \sigma \leq \tau \circ \eta \leq$ $M \sigma$ for some positive constants $m$ and $M$. Define

$$
\left(\eta^{*} f\right)(y)=f(\eta(y))\left(f \in C_{00}(X, \tau), y \in Y\right) .
$$

In this case, we see easily that $\eta^{*}$ is a homomorphism from $C_{00}(X, \tau)$ to $C_{0}(Y)$. We shall show that $\eta^{*}\left(C_{00}(X, \tau)\right)=C_{00}(Y, \sigma)$. To do this, let us take $f \in C_{00}(X, \tau)$ arbitrarily. It is clear that $\eta^{*} f \in C_{0}(Y)$. Moreover, the inequality

$$
\left|\left(\eta^{*} f\right)(y)\right| \sigma(y) \leq|f(\eta(y))| \tau(\eta(y)) / m(y \in Y),
$$

implies that $\left(\eta^{*} f\right) \sigma \in C_{0}(Y)$ since $f \tau \in C_{0}(X)$, and so $\eta^{*} f \in C_{00}(Y, \sigma)$. Namely, we obtain $\eta^{*}\left(C_{00}(X, \tau)\right) \subseteq C_{00}(Y, \sigma)$. To show the opposite inclusion, for $g \in C_{00}(Y, \sigma)$, let us define

$$
f(x)=g\left(\eta^{-1}(x)\right)(x \in X) .
$$

It is clear that $f \in C_{0}(X)$. Moreover, the inequality

$$
|f(x)| \tau(x) \leq\left|g\left(\eta^{-1}(x)\right)\right| M \sigma\left(\eta^{-1}(x)\right)(x \in X)
$$

implies that $f \tau \in C_{0}(X)$ since $g \sigma \in C_{0}(Y)$, and so $f \in C_{00}(X, \tau)$. Moreover, since

$$
\left(\eta^{*} f\right)(y)=f(\eta(y))=g\left(\eta^{-1}(\eta(y))\right)=g(y)(y \in Y)
$$

holds, we have

$$
\eta^{*} f=g,
$$

namely, we obtain $C_{00}(Y, \sigma) \subseteq \eta^{*}\left(C_{00}(X, \tau)\right)$. Therefore, we have the desired equality. By (3.4), (3.5) and (3.6), we have

$$
m\left\|\eta^{*} f\right\|_{\infty, \sigma} \leq\|f\|_{\infty, \tau} \leq M\left\|\eta^{*} f\right\|_{\infty, \sigma}\left(f \in C_{00}(X, \tau)\right),
$$

and hence $\eta^{*}$ is an isomorphism from $C_{00}(X, \tau)$ onto $C_{00}(Y, \sigma)$.

(ii) $\Rightarrow$ (iii) This can be shown in the same manner as the proof of (i) $\Rightarrow$ (iii).

(iii) $\Rightarrow$ (ii) This can be shown in the same manner as the proof of (iii) $\Rightarrow$ (i).

This completes the proof of Theorem 1.1.

Corollary 3.1. The following four statements are equivalent:

(i) $C_{0 b}(X, \tau)$ is of BSE and of BED.

(ii) $\tau$ is bounded.

(iii) $C_{0 b}(X, \tau)=C_{0}(X)$.

(iv) $C_{0 b}(X, \tau)$ is isomorphic to some commutative $C^{*}$-algebra. 
Proof. (i) $\Leftrightarrow$ (iv) This immediately follows from Lemma 2.2 and [2, Corollary 4.2].

(ii) $\Rightarrow$ (iii) Obvious.

(iii) $\Rightarrow$ (iv) Obvious.

(iv) $\Rightarrow$ (ii) Suppose that $C_{0 b}(X, \tau)$ is isomorphic to $C_{0}(Y)$ for some locally compact Hausdorff space $Y$ and define

$$
1_{Y}(y)=1(y \in Y) .
$$

Then $C_{0 b}(X, \tau)$ is isomorphic to $C_{0 b}\left(Y, 1_{Y}\right)$ since $C_{0 b}\left(Y, 1_{Y}\right)=C_{0}(Y)$. By Theorem 1.1, we can find a homeomorphism $\eta$ from $Y$ onto $X$ such that $m 1_{Y} \leq \tau \circ \eta \leq M 1_{Y}$ for some positive constants $m$ and $M$. Therefore, we have that $\tau(x) \leq M$ for all $x \in X$.

Corollary 3.2. The following three statements are equivalent:

(i) $\tau$ is unbounded.

(ii) $C_{0 b}(X, \tau)$ has no bounded $X$-weak approximate identity.

(iii) $C_{0 b}(X, \tau)$ is not of $B S E$.

Proof. (i) $\Rightarrow$ (ii) Suppose that $\tau$ is unbounded. If $C_{0 b}(X, \tau)$ has a bounded $X$-weak approximate identity, say, $\left\{e_{\lambda}\right\}_{\lambda \in \Lambda}$ bounded by $\beta$, then we can take $x_{0} \in X$ and $\lambda_{0} \in \Lambda$ such that $\tau\left(x_{0}\right) \geq$ $2 \beta+1$ and $\left|e_{\lambda_{0}}\left(x_{0}\right)-1\right| \leq 1 / 2$ since $\tau$ is unbounded. Then we have

$$
\beta \geq\left\|e_{\lambda_{0}}\right\|_{\infty, \tau}=\sup _{x \in X}\left|e_{\lambda_{0}}(x)\right| \tau(x) \geq\left|e_{\lambda_{0}}\left(x_{0}\right)\right| \tau\left(x_{0}\right) \geq \frac{2 \beta+1}{2}=\beta+\frac{1}{2},
$$

which is a contradiction.

(ii) $\Rightarrow$ (iii) This immediately follows from [13, Corollary 5].

(iii) $\Rightarrow$ (i) This immediately follows from Corollary 3.1 since an arbitrary commutative $C^{*}$ algebra is of BSE (see [13, Theorem 3]).

\section{Proof of Theorem 1.2}

Let $Y$ be another locally compact Hausdorff space and $\sigma$ be another positive continuous function on $Y$ with $\inf _{y \in Y} \sigma(y) \geq 1$.

(i) $\Rightarrow$ (ii) Suppose that there is an isomorphism $\rho$ from $C_{00}(X, \tau)$ onto $C_{0 b}(Y, \sigma)$. Let $\rho^{*}$ be the dual map of $\rho$ from $C_{0 b}(Y, \sigma)^{*}$ onto $C_{00}(X, \tau)^{*}$, and then we have $\rho^{*}\left(\Phi_{C_{0 b}(Y, \sigma)}\right)=\Phi_{C_{00}(X, \tau)}$. Let $\delta_{X}$ and $\delta_{Y}$ be the natural embedding of $X$ onto $\Phi_{C_{00}(X, \tau)}$ and the natural embedding of $Y$ onto $\Phi_{C_{0 b}(Y, \sigma)}$, respectively. Define

$$
\eta=\left.\left(\delta_{X}\right)^{-1} \circ \rho^{*}\right|_{\Phi_{C_{0 b}(Y, \sigma)}} \circ \delta_{Y} .
$$

Then $\eta$ is a homeomorphism from $Y$ onto $X$. Moreover, as observed in the proof of (i) $\Rightarrow$ (iii) in Theorem 1.1, the equality $\rho(f)=f \circ \eta$ holds for all $f \in C_{00}(X, \tau)$ and there are positive constants $m$ and $M$ such that $m \sigma \leq \tau \circ \eta \leq M \sigma$. Define

$$
\left(\eta^{*} f\right)(y)=f(\eta(y))\left(f \in C_{00}(X, \tau), y \in Y\right) .
$$

Then, as observed in the proof of (iii) $\Rightarrow(\mathrm{i})$ in Theorem 1.1 , the equality $\eta^{*}\left(C_{00}(X, \tau)\right)$ $=C_{00}(Y, \sigma)$ holds. However since $\rho=\eta^{*}$, it follows that

$$
C_{0 b}(Y, \sigma)=\rho\left(C_{00}(X, \tau)\right)=\eta^{*}\left(C_{00}(X, \tau)\right)=C_{00}(Y, \sigma),
$$

and hence we have

$$
C_{00}(Y, \sigma)=C_{0 b}(Y, \sigma) .
$$

Assume that $\sigma$ is unbounded. Then we can find a sequence $\left\{y_{1}, y_{2}, \cdots\right\}$ in $Y$ and a sequence $\left\{n_{1}, n_{2}, \cdots\right\}$ in $\mathbf{N}$ such that

$$
n_{1}<\sigma\left(y_{1}\right)<n_{2}<\sigma\left(y_{2}\right)<n_{3}<\sigma\left(y_{3}\right)<n_{4}<\cdots \cdots,
$$


where $n_{1}=1$. In this case, $\lim _{n \rightarrow \infty} y_{n}=\omega_{Y}$ holds, where $\omega_{Y}$ is the point of $Y$ at infinity. In fact, let $K$ be an arbitrary compact subset of $Y$ and put $\alpha_{K}=\max \{\sigma(y): y \in K\}$. Take $i_{0} \in \mathbf{N}$ with $\alpha_{K}<n_{i_{0}}$. Then we can easily see that $y_{j} \in Y \backslash K$ for all $j \geq i_{0}$, that is, $\lim _{n \rightarrow \infty} y_{n}=\omega_{Y}$.

Take $m \in \mathbf{N}$ arbitrarily. Since $\sigma$ is continuous on $Y$, we can find a compact neighbourhood $K_{m}$ of $y_{m}$ such that $K_{m} \subseteq\left\{y \in Y: n_{m}<\sigma(y)<n_{m+1}\right\}$ and $\left|\sigma\left(y_{m}\right)-\sigma(y)\right|<1\left(y \in K_{m}\right)$. Then $K_{i} \cap K_{j}=\emptyset(i \neq j)$. Take a continuous positive function $g_{m}$ on Y such that

$$
g_{m}\left(y_{m}\right)=\frac{1}{\sigma\left(y_{m}\right)}, 0 \leq g_{m} \leq \frac{1}{\sigma\left(y_{m}\right)} \text { and } \operatorname{supp}\left(g_{m}\right) \subseteq K_{m} .
$$

Since $\left|1-\frac{\sigma(y)}{\sigma\left(y_{m}\right)}\right|<1 / \sigma\left(y_{m}\right) \leq 1$ for all $y \in K_{m}$, it follows that

$$
0 \leq g_{m}(y) \sigma(y) \leq \frac{\sigma(y)}{\sigma\left(y_{m}\right)} \leq 2\left(y \in K_{m}\right) .
$$

Define

$$
g(y)=\sum_{m=1}^{\infty} g_{m}(y)(y \in Y) .
$$

Then it is clear that $g$ is continuous on $X$ such that $0 \leq g \sigma \leq 2$, and hence $g \sigma$ is bounded. Moreover, we shall show that $g \in C_{0}(Y)$. To do this, let $\varepsilon$ be an arbitrary positive number. Take $j_{0} \in \mathbf{N}$ with $1 / n_{j_{0}}<\varepsilon$ and put

$$
K_{0}=K_{1} \cup K_{2} \cup \cdots \cup K_{n_{j_{0}}} .
$$

Then $K_{0}$ is a compact set in $Y$. Take $y \in Y \backslash K_{0}$ arbitrarily. Then we have two cases:

(a) $y \notin \cup_{i=1}^{\infty} K_{i}$

and

(b) $y \in K_{k_{0}}$ for some $k_{0}>n_{j_{0}}$.

In case (a), we have $g(y)=0<\varepsilon$. In case (b), we have

$$
0 \leq g(y)=g_{k_{0}}(y) \leq 1 / \sigma\left(y_{k_{0}}\right)<1 / n_{k_{0}} \leq 1 / k_{0}<1 / n_{j_{0}}<\varepsilon .
$$

Then $g \in C_{0}(Y)$ as required. Thus we get $g \in C_{0 b}(Y, \sigma)$. Therefore, it follows from (4.1) that $g \in C_{00}(Y, \sigma)$, and hence $\lim _{n \rightarrow \infty} g\left(y_{n}\right) \sigma\left(y_{n}\right)=0$. But since $g\left(y_{n}\right) \sigma\left(y_{n}\right)=g_{n}\left(y_{n}\right) \sigma\left(y_{n}\right)=1$ holds for all $n \in \mathbf{N}$, we arrive at a contradiction. Hence we conclude that $\sigma$ is bounded. This implies that $\tau$ is also bounded because $\tau \circ \eta \leq M \sigma$.

(ii) $\Rightarrow$ (i) Suppose that both $\tau$ and $\sigma$ are bounded. Then we see that $C_{00}(X, \tau)=C_{0}(X)$ and $C_{0 b}(Y, \sigma)=C_{0}(Y)$. If $X$ is homeomorphic to $Y$, then $C_{0}(X)$ is isomorphic to $C_{0}(Y)$, and hence $C_{00}(X, \tau)$ must be isomorphic to $C_{0 b}(Y, \sigma)$.

This completes the proof of Theorem 1.2.

Corollary 4.3. The following two statements are equivalent:

(i) $C_{0 b}(X, \tau)$ is Tauberian.

(ii) $\tau$ is bounded.

Proof. (i) $\Rightarrow$ (ii) Suppose that $C_{0 b}(X, \tau)$ is Tauberian. Then $C_{00}(X, \tau)=C_{0 b}(X, \tau)$ holds. In fact, take $f \in C_{0 b}(X, \tau)$ and $\varepsilon>0$ arbitrarily. Then $f \in C_{0}(X)$. Also since $C_{0 b}(X, \tau)$ is Tauberian, it follows that there is $g \in C_{c}(X)$ with $\|f-g\|_{\infty, \tau}<\varepsilon$. Therefore, $g \tau \in C_{c}(X)$ and $\|f \tau-g \tau\|_{\infty}=$ $\|f-g\|_{\infty, \tau}<\varepsilon$, and hence $f \tau \in C_{0}(X)$ because $\varepsilon$ is arbitrary. Thus we have $f \in C_{00}(X, \tau)$, namely, $C_{00}(X, \tau)=C_{0 b}(X, \tau)$ holds as required. Consequently, $C_{00}(X, \tau)$ must be isomorphic to $C_{0 b}(X, \tau)$, and hence $\tau$ must be bounded by Theorem 1.2.

(ii) $\Rightarrow$ (i) Suppose that $\tau$ is bounded. Then we have $C_{0 b}(X, \tau)=C_{0}(X)$, and hence $C_{0 b}(X, \tau)$ must be Tauberian. 


\section{BSE-EXTENSION AND INOUE-DOSS IDEAL}

Let $A$ be $C_{00}(X, \tau)$ or $C_{0 b}(X, \tau)$. In this section, we investigate the BSE-extension associated with $A$ and the Inoue-Doss ideal associated with $A$. The obtained result is as follows:

\section{Theorem 5.4.}

(i) The BSE-extensions associated with $C_{00}(X, \tau)$ and $C_{0 b}(X, \tau)$ are both equal to $C_{b b}(X, \tau)$, that is,

$$
C_{B S E\left(C_{00}(X, \tau)\right)}(X)=C_{B S E\left(C_{0 b}(X, \tau)\right)}(X)=C_{b b}(X, \tau) .
$$

(ii) The Inoue-Doss ideals associated with $C_{00}(X, \tau)$ and $C_{0 b}(X, \tau)$ are both equal to $C_{00}(X, \tau)$, that is,

$$
C_{B S E\left(C_{00}(X, \tau)\right)}^{0}(X)=C_{B S E\left(C_{0 b}(X, \tau)\right)}^{0}(X)=C_{00}(X, \tau) .
$$

Proof. Denote by $A$ any one of the algebras $C_{00}(X, \tau)$ and $C_{0 b}(X, \tau)$, and then $A \subseteq C_{c}(X)$.

(i) We shall show $C_{B S E(A)}(X)=C_{b b}(X, \tau)$. To do this, we claim that

$$
\|p\|_{A^{*}}=\sum_{x \in X}|\widehat{p}(x)| / \tau(x)(p \in \operatorname{span}(X))
$$

holds. In fact, let us take $p \in \operatorname{span}(X)$ and $0<\varepsilon<1$ arbitrarily. Then we can write $p=$ $\sum_{k=1}^{n} a_{k} x_{k}$, where $a_{1}, \cdots, a_{n} \in \mathbf{C} \backslash\{0\}$ and $x_{1}, \cdots, x_{n} \in X$ with $x_{i} \neq x_{j}(i \neq j)$. For each $1 \leq k \leq n$, we can take a compact neighbourhood $K_{k}$ of $x_{k}$ such that $K_{i} \cap K_{j}=\emptyset(i \neq j)$ and $(1-\varepsilon) / \tau\left(x_{k}\right)<1 / \tau(x)$ for all $x \in K_{k}$ because

$$
\left\{x \in X: \frac{1-\varepsilon}{\tau\left(x_{k}\right)}<\frac{1}{\tau(x)}\right\}
$$

is an open neighbourhood of $x_{k}$. Take a continuous positive function $g_{k}$ on $\mathrm{X}$ such that

$$
g_{k}\left(x_{k}\right)=\frac{1-\varepsilon}{\tau\left(x_{k}\right)}, 0 \leq g_{k}(x) \leq \frac{1-\varepsilon}{\tau\left(x_{k}\right)}\left(x \in K_{k}\right) \text { and } \operatorname{supp}\left(g_{k}\right) \subseteq K_{k},
$$

and define

$$
g_{0}(x)=\sum_{k=1}^{n} g_{k}(x)(x \in X) .
$$

Therefore, we can easily show that $g_{0} \in C_{c}(X), g_{0}\left(x_{k}\right)=(1-\varepsilon) / \tau\left(x_{k}\right)(1 \leq k \leq n)$ and $0 \leq g_{0}(x) \leq 1 / \tau(x)(x \in X)$. Moreover, we can find a function $h_{0} \in C_{c}(X)$ such that $\left\|h_{0}\right\|_{\infty}=1$ and $h_{0}\left(x_{k}\right)=\left|a_{k}\right| / a_{k}$ for all $1 \leq k \leq n$. Put $f_{0}=g_{0} h_{0}$. Then we can see that $f_{0} \in A$, $\left\|f_{0}\right\|_{\infty, \tau} \leq 1,\left|f_{0}\left(x_{k}\right)\right| \tau\left(x_{k}\right)=1-\varepsilon$ and $a_{k} f_{0}\left(x_{k}\right)>0$ for all $1 \leq k \leq n$. Therefore, we have

$$
\begin{aligned}
\|p\|_{A^{*}} & =\sup \left\{\left|\sum_{k=1}^{n} a_{k} f\left(x_{k}\right)\right|: f \in A,\|f\|_{\infty, \tau} \leq 1\right\} \\
& \geq \sum_{k=1}^{n} a_{k} f_{0}\left(x_{k}\right)=\sum_{k=1}^{n}\left|a_{k}\right| \tau\left(x_{k}\right)^{-1}\left|f_{0}\left(x_{k}\right)\right| \tau\left(x_{k}\right) \\
& =(1-\varepsilon) \sum_{k=1}^{n}\left|a_{k}\right| / \tau\left(x_{k}\right)=(1-\varepsilon) \sum_{x \in X}|\widehat{p}(x)| / \tau(x),
\end{aligned}
$$

and hence $\|p\|_{A^{*}} \geq \sum_{x \in X}|\widehat{p}(x)| / \tau(x)$ because $\varepsilon$ is arbitrary. 
On the other hand, we have

$$
\begin{aligned}
\|p\|_{A^{*}} & =\sup \left\{\left|\sum_{k=1}^{n} a_{k} f\left(x_{k}\right)\right|: f \in A,\|f\|_{\infty, \tau} \leq 1\right\} \\
& \leq \sup \left\{\sum_{k=1}^{n}\left|a_{k}\right| \tau\left(x_{k}\right)^{-1}\left|f\left(x_{k}\right)\right| \tau\left(x_{k}\right): f \in A,\|f\|_{\infty, \tau} \leq 1\right\} \\
& \leq \sum_{k=1}^{n}\left|a_{k}\right| / \tau\left(x_{k}\right)=\sum_{x \in X}^{n}|\widehat{p}(x)| / \tau(x) .
\end{aligned}
$$

Consequently, we have proved (5.1).

Now, by (5.1), for all $f \in C_{b b}(X, \tau)$, we have

$$
\begin{aligned}
\|f\|_{B S E(A)} & =\sup \left\{\left|\sum_{x \in X} \widehat{p}(x) f(x)\right|: p \in \operatorname{span}(X),\|p\|_{A^{*}} \leq 1\right\} \\
& \leq \sup \left\{\sum_{x \in X}|\widehat{p}(x)| \tau(x)^{-1}|f(x)| \tau(x): p \in \operatorname{span}(X),\|p\|_{A^{*}} \leq 1\right\} \\
& \leq\|f\|_{\infty, \tau} \sup \left\{\sum_{x \in X}|\widehat{p}(x)| / \tau(x): p \in \operatorname{span}(X),\|p\|_{A^{*}} \leq 1\right\} \\
& =\|f\|_{\infty, \tau} \sup \left\{\|p\|_{A^{*}}: p \in \operatorname{span}(X),\|p\|_{A^{*}} \leq 1\right\} \quad(\text { by }(5.1)) \\
& =\|f\|_{\infty, \tau}<\infty,
\end{aligned}
$$

that is, $f \in C_{B S E(A)}(X)$. Therefore, we have $C_{b b}(X, \tau) \subseteq C_{B S E(A)}(X)$. To show the opposite inclusion, take $f \in C_{B S E(A)}(X)$ arbitrarily. Then $f \in C_{b}(X)$. For each $x \in X$, put $p_{x}=\tau(x) x$, that is, $p_{x}(f)=\tau(x) f(x)(f \in A)$. Then we have

$$
\begin{aligned}
\left\|p_{x}\right\|_{A^{*}} & =\sup \left\{\left|p_{x}(f)\right|: f \in A,\|f\|_{\infty, \tau} \leq 1\right\} \\
& =\sup \left\{|f(x)| \tau(x): f \in A,\|f\|_{\infty, \tau} \leq 1\right\} \\
& \leq 1
\end{aligned}
$$

holds for all $x \in X$. Therefore, we have

$$
|f(x) \tau(x)|=\left|\widehat{p}_{x}(x) f(x)\right| \leq\|f\|_{B S E(A)}
$$

for all $x \in X$, and hence $\|f \tau\|_{\infty} \leq\|f\|_{B S E(A)}<\infty$, so $f \tau \in C_{b}(X)$, that is, $f \in C_{b b}(X, \tau)$. Therefore, we have $C_{B S E(A)}(X) \subseteq C_{b b}(X, \tau)$. This completes the proof of the equality $C_{B S E(A)}(X)=$ $C_{b b}(X, \tau)$.

(ii) Let $f \in C_{b b}(X, \tau)$ and $K \in \mathcal{K}(X)$. Since

$$
\begin{aligned}
& \|f\|_{B S E(A), K}=\sup \left\{\left|\sum_{x \in X \backslash K} \widehat{p}(x) f(x)\right|: p \in \operatorname{span}(X),\|p\|_{A^{*}} \leq 1\right\} \\
& \leq \sup \left\{\sum_{x \in X \backslash K}|\widehat{p}(x)| \tau(x)^{-1}|f(x)| \tau(x): p \in \operatorname{span}(X),\|p\|_{A^{*}} \leq 1\right\} \\
& \leq \sup _{x \in X \backslash K}|f(x)| \tau(x) \times \sup \left\{\sum_{x \in X \backslash K}|\widehat{p}(x)| / \tau(x): p \in \operatorname{span}(X),\|p\|_{A^{*}} \leq 1\right\}
\end{aligned}
$$




$$
\begin{aligned}
& \leq \sup _{x \in X \backslash K}|f(x)| \tau(x) \times \sup \left\{\sum_{x \in X}|\widehat{p}(x)| / \tau(x): p \in \operatorname{span}(X),\|p\|_{A^{*}} \leq 1\right\} \\
& =\sup _{x \in X \backslash K}|f(x)| \tau(x) \times \sup \left\{\|p\|_{A^{*}}: p \in \operatorname{span}(X),\|p\|_{A^{*}} \leq 1\right\} \quad(\text { by }(5.1)) \\
& =\sup _{x \in X \backslash K}|f(x)| \tau(x),
\end{aligned}
$$

it follows that

$$
\|f\|_{B S E(A), K} \leq \sup _{x \in X \backslash K}|f(x)| \tau(x) .
$$

To show the reverse of the above inequality, put $p_{x}=\tau(x) x$ for each $x \in X$. Then we have $\left\|p_{x}\right\|_{A^{*}} \leq 1(x \in X)$ as observed in the proof of (i). Then we have

$$
|f(x) \tau(x)|=\left|\widehat{p}_{x}(x) f(x)\right| \leq\|f\|_{B S E(A), K}(x \in X \backslash K),
$$

and hence

$$
\sup _{x \in X \backslash K}|f(x)| \tau(x) \leq\|f\|_{B S E(A), K}
$$

Therefore, we have

$$
\|f\|_{B S E\left(C_{00}(X, \tau)\right), K}=\|f\|_{B S E\left(C_{0 b}(X, \tau)\right), K}\left(f \in C_{b b}(X, \tau), K \in \mathcal{K}(X)\right) .
$$

Hence it follows from (i) and (5.2) that

$$
C_{B S E\left(C_{00}(X, \tau)\right)}^{0}(X)=C_{B S E\left(C_{0 b}(X, \tau)\right)}^{0}(X) .
$$

Recall that $C_{00}(X, \tau)$ is of BED, and hence $C_{B S E\left(C_{00}(X, \tau)\right)}^{0}(X)=C_{00}(X, \tau)$ holds.

\section{Remark 5.2.}

(i) If $\tau$ is bounded, then $C_{00}(X, \tau)=C_{0 b}(X, \tau)=C_{0}(X)$ and $C_{b b}(X, \tau)=C_{b}(X)$, and hence Theorem 5.4 obviously holds.

(ii) As observed in the proof of Theorem 5.4 (i),

$$
\|f\|_{B S E\left(C_{00}(X, \tau)\right)}=\|f\|_{B S E\left(C_{0 b}(X, \tau)\right)}=\|f\|_{\infty, \tau}
$$

holds for all $f \in C_{b b}(X, \tau)$.

(iii) As observed in the proof of Theorem 5.4 (ii),

$$
\|f\|_{B S E\left(C_{00}(X, \tau)\right), K}=\|f\|_{B S E\left(C_{0 b}(X, \tau)\right), K}=\sup _{x \in X \backslash K} \mid f(x) \tau(x)
$$

holds for all $f \in C_{b b}(X, \tau)$ and $K \in \mathcal{K}(X)$.

Corollary 5.4. If $\tau$ is unbounded, then $C_{0 b}(X, \tau)$ is not of $B E D$.

Proof. Suppose that $\tau$ is unbounded. Then $C_{00}(X, \tau) \varsubsetneqq C_{0 b}(X, \tau)$ holds. In fact, suppose on the contrary that $C_{00}(X, \tau)=C_{0 b}(X, \tau)$ holds. Then $C_{00}(X, \tau)$ is isomorphic to $C_{0 b}(X, \tau)$, and hence $\tau$ must be bounded by Theorem 1.2. This is impossible because $\tau$ is unbounded by hypothesis. Now if $C_{0 b}(X, \tau)$ is of BED, then we have from Theorem 5.4 (ii) that

$$
\left.C_{00}(X, \tau)=C_{B S E\left(C_{00}(X, \tau)\right)}^{0}(X)=C_{B S E\left(C_{0 b}(X, \tau)\right)}^{0}(X)=C_{0 b}(X, \tau)\right) .
$$

Thus we arrive at a contradiction. 


\section{Proof of Theorem 1.3}

We can see that:

(1) $(\mathrm{v}) \Leftrightarrow(\mathrm{iv}) \Leftrightarrow(\mathrm{i})$ are derived from Corollary 3.2.

(2) $(\mathrm{v}) \Leftrightarrow$ (iii) is exactly the same as Corollary 4.3.

(3) (ii) $\Leftrightarrow(\mathrm{v})$ is derived from Corollary 5.4.

By combining (1), (2) and (3), we have proved Theorem 1.3.

Remark 6.3. The following four statements are equivalent:

(i) $\tau$ is bounded.

(ii) $C_{00}(X, \tau)$ is isomorphic to some commutative $C^{*}$-algebra.

(iii) $C_{0 b}(X, \tau)$ is isomorphic to some commutative $C^{*}$-algebra.

(iv) $C_{b b}(X, \tau)$ is isomorphic to some commutative $C^{*}$-algebra.

In fact, since $C_{0 b}(X, \tau)$ is a closed ideal of $C_{b b}(X, \tau)$, it follows that (iv) implies (iii). Also since $C_{00}(X, \tau)$ is a closed ideal of $C_{0 b}(X, \tau)$, it follows that (iii) implies (ii). If $C_{00}(X, \tau)$ is isomorphic to some commutative $C^{*}$-algebra, then it must be of BSE, and hence $M\left(C_{00}(X, \tau)\right)=$ $C_{B S E\left(C_{00}(X, \tau)\right)}(X)$. Moreover, we have from Theorem $5.4(\mathrm{i})$ that $C_{B S E\left(C_{00}(X, \tau)\right)}(X)=C_{b b}(X, \tau)$, and hence $M\left(C_{00}(X, \tau)\right)=C_{b b}(X, \tau)$. Define $1_{X}(x)=1(x \in X)$. Then $1_{X} \in M\left(C_{00}(X, \tau)\right)$, and hence $1_{X} \in C_{b b}(X, \tau)$ by the above equality. Then $\tau$ must be bounded. Consequently, (ii) implies (i). If $\tau$ is bounded, then $C_{b b}(X, \tau)=C_{b}(X)$, and so $C_{b b}(X, \tau)$ is isomorphic to the $\mathrm{C}^{*}$-algebra $C_{b}(X)$. Then (i) implies (iv).

\section{EXAMPLES}

Let $\mathbf{R}$ be the space of real numbers with usual topology and Homeo( $\mathbf{R})$ be the set of all homeomorphisms from $\mathbf{R}$ onto itself. Let $\mathcal{T}(\mathbf{R})$ be the set of all positive continuous functions $\tau$ on $\mathbf{R}$ with $\inf \{\tau(x): x \in \mathbf{R}\} \geq 1$. Let $\tau, \sigma \in \mathcal{T}(\mathbf{R})$. If there are $m, M>0$ and $h \in$ Homeo $(\mathbf{R})$ such that $m \tau \leq \sigma \circ h \leq M \tau, \tau$ and $\sigma$ are said to be equivalent, and written as $\tau \cong \sigma$.

(i) Take $\tau \in \mathcal{T}(\mathbf{R})$ and $h \in H o m e o(\mathbf{R})$ arbitrarily. Then we have $\tau \cong \tau \circ h$ because $\tau=(\tau \circ h) \circ h^{-1}$.

(ii) Define

$$
\tau_{1}(x)= \begin{cases}(n+1)(|x|-2 n)+1 & (2 n \leq|x| \leq 2 n+1) \\ -(n+1)(|x|-2 n-2)+1 & (2 n+1<|x| \leq 2 n+2),\end{cases}
$$

where $n=0,1,2, \cdots$. Then $\tau_{1} \in \mathcal{T}(\mathbf{R})$. Since $\tau_{1}$ is unbounded, it follows from Corollary 4.3 that $C_{0 b}\left(\mathbf{R}, \tau_{1}\right)$ is not Tauberian. However, we can confirm this by a concrete calculation as follows. Define

$$
f(x)=\frac{1}{\tau_{1}(x) \tau_{1}(x-1)}(x \in \mathbf{R}) .
$$

Then we can easily see $f \in C_{0 b}\left(\mathbf{R}, \tau_{1}\right)$. Also since $\tau_{1}(2 n)=1$ for all $n=0, \pm 1, \pm 2, \cdots$, it follows that

$$
\|f-g\|_{\infty, \tau_{1}}=\sup _{x \in \mathbf{R}}\left|\frac{1}{\tau_{1}(x-1)}-g(x) \tau_{1}(x)\right| \geq 1
$$

for all $g \in C_{c}(\mathbf{R})$. In other words, $C_{0 b}\left(\mathbf{R}, \tau_{1}\right)$ is not Tauberian.

(iii) Define

$$
\tau_{0}(x)=1+|x|(x \in \mathbf{R}) .
$$

Then $\tau_{0} \in \mathcal{T}(\mathbf{R})$ and we can easily see $\tau_{0} \nsubseteq \tau_{1}$. Therefore, $C_{0 b}\left(\mathbf{R}, \tau_{0}\right)$ is not isomorphic to $C_{0 b}\left(\mathbf{R}, \tau_{1}\right)$ by Theorem 1.1. 
(iv) Let $f$ be a strictly increasing continuous function on $[0, \infty)$ such that $f(0)=0$ and $\lim _{x \rightarrow+\infty} f(x)=+\infty$. Let $g$ be a strictly decreasing continuous function on $(-\infty, 0]$ such that $g(0)=0$ and $\lim _{x \rightarrow-\infty} g(x)=+\infty$. Define

$$
\tau_{f, g}(x)=\left\{\begin{array}{ll}
1+f(x) & (x \geq 0) \\
1+g(x) & (x<0)
\end{array} .\right.
$$

Then we see $\tau_{f, g} \in \mathcal{T}(\mathbf{R})$ and $\tau_{0} \cong \tau_{f, g}$. In fact, it is clear that $\tau_{f, g} \in \mathcal{T}(\mathbf{R})$. Define

$$
h(x)=\left\{\begin{array}{ll}
f^{-1}(x) & (x \geq 0) \\
g^{-1}(-x) & (x<0)
\end{array} .\right.
$$

Then we see $h \in$ Homeo $(\mathbf{R})$ and $\tau_{f, g} \circ h=\tau_{0}$ by an easy calculation. Therefore, we obtain $\tau_{f, g} \cong \tau_{0}$ from (i), and hence $C_{0 b}\left(\mathbf{R}, \tau_{f, g}\right)$ is isomorphic to $C_{0 b}\left(\mathbf{R}, \tau_{0}\right)$ by Theorem 1.1.

\section{OPEN PROBLEMS}

Finally, let us list some open problems for further study.

Problem on vector-valued functions: Let $X$ be a locally compact Hausdorff space, $\tau$ be a positive continuous function on $X$ with $\inf _{x \in X} \tau(x) \geq 1$ and $A$ be a unital commutative $C^{*}$-algebra. Moreover, let $C_{0}(X, A)$ be the commutative Banach algebra of all continuous $A$-valued functions on $X$ vanishing at infinity and $C_{b}(X, A)$ be the commutative Banach algebra of all bounded continuous $A$-valued functions. Define

$$
C_{0 b}(X ; A, \tau)=\left\{f \in C_{0}(X, A): \tau f \in C_{b}(X, A)\right\} .
$$

Then, solve the isomorphism problem in the Banach algebra $C_{0 b}(X ; A, \tau)$.

Moreover, what are the BSE-extension and the Inoue-Doss ideal associated with $C_{0 b}(X ; A, \tau)$ ?

Problem on Lipschitz algebras: $\operatorname{Let}_{\operatorname{Lip}_{1}}(\mathbf{R})$ and $\operatorname{Lip}_{1}^{0}(\mathbf{R})$ be the Lipschitz algebras as defined in [4, Definition 5.8] and $\tau$ be a positive continuous function on $\mathbf{R}$ with $\inf _{x \in X} \tau(x) \geq 1$. Define

$$
\operatorname{Lip}_{01}(\mathbf{R}, \tau)=\left\{f \in \operatorname{Lip}_{1}^{0}(\mathbf{R}): \tau f \in \operatorname{Lip}_{1}(\mathbf{R})\right\} .
$$

Then, what are the BSE-extension and the Inoue-Doss ideal associated with the Banach algebra $\operatorname{Lip}_{01}(\mathbf{R}, \tau)$ ?

Problem on differentiable functions: Let $C_{b}^{n}\left(\mathbf{R}^{d}\right)$ and $C_{0}^{n}\left(\mathbf{R}^{d}\right)$ be the differential algebras as defined in $[7, \S 2]$ and $\tau$ be a positive continuous function on $\mathbf{R}$ with $\inf _{x \in X} \tau(x) \geq$ 1. Define

$$
C_{01}^{n}\left(\mathbf{R}^{d}, \tau\right)=\left\{f \in C_{0}^{n}\left(\mathbf{R}^{d}\right): \tau f \in C_{b}^{n}\left(\mathbf{R}^{d}\right)\right\} .
$$

Then, what are the BSE-extension and the Inoue-Doss ideal associated with the Banach algebra $C_{01}^{n}\left(\mathbf{R}^{d}, \tau\right)$ ?

\section{ACKNOWLEDGEMENTS}

This work was supported by the Research Institute for Mathematical Sciences, a Joint Usage/Research Center located in Kyoto University.

The authors are grateful to the referees for valuable suggestions and comments. 


\title{
REFERENCES
}

[1] R. Doss: On the transform of a singular or an absolutely continuous measure, Proc. Amer. Math. Soc., 19 (1968), 361-363.

[2] J. Inoue, T. Miura, H. Takagi and S.-E. Takahasi: Classification of semisimple commutative Banach algebras of type I, Nihonkai Math. J., 30 (1) (2019), 1-17.

[3] J. Inoue, S.-E. Takahasi: Constructions of bounded weak approximate identities for Segal algebras on LCA groups, Acta Sci. Math., 66 (2000), 257-271.

[4] J. Inoue, S.-E. Takahasi: On characterizations of the image of the Gelfand transform of commutative Banach algebras, Math. Nachr., 280 (2007), 105-126.

[5] J. Inoue, S.-E. Takahasi: Segal algebras in commutative Banach algebras, Rocky Mountain J. Math., 44 (2) (2014), 539-589.

[6] J. Inoue, S.-E. Takahasi: A construction of a BSE-algebra of type I which is isomorphic to no $C^{*}$-algebras, Rocky Mountain J. Math., 47 (8) (2017), 2693-2697.

[7] J. Inoue, S.-E. Takahasi: Banach function algebras of n-times continuously differentiable functions on $\mathbb{R}^{d}$ vanishing at infinity and their BSE-extensions, J. Korean Math. Soc., 56 (5) (2019), 1333-1354.

[8] C. A. Jones, C. D. Lahr: Weak and norm approximate identities are different, Pac. J. Math., 72 (1977), 99-104.

[9] E. Kaniuth, A. Ülger: The Bochner-Schoenberg-Eberlein property for commutative Banach algebras, especially Fourier and Fourier Stieltjes algebras, Trans. Amer. Math. Soc., 362 (2010), 4331-4356.

[10] H. Reiter: $L^{1}$-algebras and Segal algebras, Lect. Notes Math., 231, Springer-Verlag, Berlin (1971).

[11] H. Reiter, J. D. Stegeman: Classical Harmonic Analysis and Locally compact groups, Oxford Science Publications, Oxford (2000).

[12] C. E. Rickart: General Theory of Banach Algebras, D. Van Nostrand Company, Inc. Princeton, New Jersey, Toronto, London, New York (1960).

[13] S.-E. Takahasi, O. Hatori: Commutative Banach algebras which satisfy a Bochner-Schoenberg-Eberlein type-theorem, Proc. Amer. Math. Soc., 110 (1) (1990), 149-158.

\author{
SIN-EI TAKAHASI \\ LABORATORY OF MATHEMATICS AND GAMES \\ KATSUSHIKA 2-371, FUNABASHI, CHIBA 273-0032, JAPAN \\ ORCID: 0000-0002-7936-2332 \\ URL: https://math-game-labo.com \\ E-mail address: sin_ei1@yahoo.co.jp \\ KIYOSHI SHIRAYANAGI \\ TOHO UNIVERSITY \\ DEPARTMENT OF INFORMATION SCIENCE \\ MIYAMA 2-2-1, FUNABASHI, CHIBA 274-8510, JAPAN \\ ORCID: 0000-0001-8123-3823 \\ E-mail address: kiyoshi.shirayanagieis.sci.toho-u.ac.jp
}

MAKOTO TSUKADA

TOHO UNIVERSITY

DEPARTMENT OF INFORMATION SCIENCE

MiYAMA 2-2-1, FUNABASHI, CHIBA 274-8510, JAPAN

ORCID: 0000-0001-5405-8900

E-mail address: tsukada@is.sci.toho-u.ac.jp 